\title{
WHAT CAN LIFE ON EARTH TELL US ABOUT LIFE IN THE UNIVERSE?
}

\author{
CHARLES H. LINEWEAVER \& ADITYA CHOPRA
}

Planetary Science Institute, Research School of Astronomy and Astrophysics and the Research School of Earth Sciences, Australian National University, Canberra, Australia

\begin{abstract}
We review the most fundamental features common to all terrestrial life. We argue that the ubiquity of these features makes them the best candidates for being features of extraterrestrial life. Other frequently espoused candidates are less secure because they are based on subjective notions of universal fitness, not on features common to all terrestrial life. For example, major transitions in the evolutionary pathway that led to Homo sapiens are sometimes considered to be fundamental transitions in the evolution of all life. However, these "major transitions" are largely arbitrary because a series of different major transitions can be identified along the evolutionary pathway to any extant species.
\end{abstract}

\section{Quirkometry and Terrestrial Life as a Model Organism}

The universe is filled with stars similar to our Sun (Robles et al., 2008), rocky planets similar to our Earth (Lineweaver and Grether, 2003; Ida and Lin 2004; Mordasini et al., 2009), water like our oceans (Kuchner, 2003; Léger et al., 2004), amino acids like our proteins and all the other ingredients for life (Pizzarello, 2007). But is the universe filled with life? If it is, what kind of life is it? We argue that if there is life out there at all, its basic features are likely to be a subset of the features common to all terrestrial life.

Here on Earth, we can distinguish generic features common to all terrestrial life from quirky features unique to only one group of organisms. Sometimes the distinction between generic and quirky is easy. For example, all terrestrial life is based on carbon, but only a tiny subset barks at passing cars. From this we can infer that on other terrestrial planets, we are more likely to find carbon-based extraterrestrials than we are to find dogs. All terrestrial life has DNA, but only a tiny subset has naked mole rat DNA. From this we can infer that on other planets, we are more likely to find extraterrestrials with DNA than we are to find naked mole rats.

Sometimes the distinction between generic and quirky is more difficult. Multicellularity, sexual reproduction and encephalization are not common to all terrestrial life. But are they common enough (or adaptive enough) that we should expect extraterrestrials to be multicellular, sexual reproducers with heads? Quirkometry - distinguishing the quirky, uniquely terrestrial, from the terrestrially generic and possibly universal - is a young science.

Biologists study model organisms such as mice (Mus musculus), fruit flys (Drosophila melanogaster), nematode roundworms (Caenorhabditis elegans), yeast 
(Saccharomyces cerevisiae), slime mold (Dictyostelium discoideum), colon bacteria (Escherichia coli), and mustard plants (Arabidopsis thaliana), not because the features of these species are more generic or representative of other life forms, or because these species are more quirky and interesting than other species, but because they are easier to study. Among the life forms in the universe, terrestrial life can be viewed as a model organism, because it is the easiest for us to study. But how representative is terrestrial life of life elsewhere?

When a Drosophila expert learns all that is known about Drosophila, the next step - the most useful step as far as other biologists are concerned - is to figure out how much of what is known about Drosophila, applies to other organisms. How generic and how quirky are the various Drosophila features? To make these distinctions, we simply compare Drosophila to many other species. But even in the absence of other species, one could make some good guesses. For example, basic Drosophila biochemistry is likely to be shared by other life forms, but the exact shape of the wings is not. Details are different from fundamentals.

Drosophila embryology can help us understand other species which had a common ancestor with Drosophila hundreds of millions of years ago. For example, segmentation occurs very early on in the embryogenesis of Drosophila and because ontogeny tends to recapitulate phylogeny (Gould, 1977) we could make an educated guess that other species, with whom Drosophila shared a common ancestor a few hundred million years ago, would also be segmented. Fundamental features of an individual member can provide information about the group because the most fundamental features appear earliest in the embryonic development of an individual and these features are often phylogenetically the most deeply rooted. Thus, they are likely to be shared by ancient ancestors and their other descendants. Similarly, the earliest "embryonic" ontogeny - the earliest steps of development of the single example of terrestrial life we know - may recapitulate the paths that all life in the universe has to follow during its earliest evolution.

Here is an example of our reasoning. Suppose we did not know that chimpanzees existed, but we wondered - or even suspected - that there were beings out there in the jungle with whom we shared a common ancestor about 6 million years ago. Without having detected chimpanzees, the best way to study these possibly-existent beings would be to study the fossils of early hominids that lived about 6 million years ago. This is because these early ancestors are (and were) more closely related to chimps than we are today. In the absence of SETI detections or visits from ET, the earliest branchings of the terrestrial phylogenetic tree of life (Figs. 3 and 4) are the closest we can get to the earliest branchings of life elsewhere. Thus, the study of the earliest and most fundamental features of terrestrial life is probably one of the best ways to study possibly-existent extraterrestrial life.

\section{Evolution: From Deterministic to Quirky}

Terrestrial life emerged from non-life. Quirky biology emerged from deterministic physics and chemistry. If this assumption is correct, then the first steps of molecular evolution are deterministic or quasi-deterministic. DeDuve (1995) has argued that this initial determinism makes life a "cosmic imperative" built into the chemistry of the universe.

The earliest life in warm little ponds or hydrothermal vents adapted to abiotic environmental challenges such as variations in temperature, $\mathrm{pH}$, salinity, solar radiation and humidity. These adaptations to environmental conditions do not have 
strong feedback. The temperature of the environment does not get higher or lower when tolerance to hotter and colder temperature evolves. The $\mathrm{pH}$ of the environment does not get higher or lower when an auto-catalytic cycle or protoorganism learns how to handle free protons.

However, as proto-life evolved into life, an increasingly important part of an organism's environment became other organisms. Life forms became dependent on each other for nutrients and metabolism. Increasingly, life had to adapt to life. Thus, evolution became more self-referential and quirky, similar to the non-linear screech of feedback when a microphone comes too close to a loudspeaker. The screech is an arbitrary random noise being amplified into a dominant signal. In a similarly positive feedback loop, DNA is pushed by selection pressure from other DNA to fitness peaks on an adaptive landscape created by the same DNA. Predator/prey arms races, sexual selection and the selective pressure from conspecifics and other life forms, provide the feedback that makes current evolution self-referential, divergent and contingent. Biology is an historical science. The further life diverges, the more its direction is determined by the accumulated quirks of past history (Gould, 1989, 2002; McShea and Brandon, 2010).

Picture a tree with a trunk that splits into branches, and branches that split into twigs. If one accepts the idea that life has evolved from a deterministic trunk into quirky twigs, and if one accepts the idea that quirkiness increases with time, then the first divergences of life, the thick branches closest to the trunk, are more likely to be representative of life elsewhere than will be the quirky twigs. Our best guesses about the nature of extraterrestrials will come from a study of the features common to all life on Earth, for it is there that the deterministic initial stages can be read. When we look at the earliest divergences in the tree of life, we are looking at divergences that took place when life was closer to its deterministic roots. In other words, the divergent paths that the early evolution of life took are likely to be more relevant to early life elsewhere, than the quirky contingent paths that life took further downstream (Fig. 3).

The most fundamental features of mice are the features they share with all mammals. The most fundamental features of mammals are the features they share with all eukaryotes. The most fundamental features of eukaryotes are the features they share with all terrestrial life. And among the most fundamental features of terrestrial life forms are the features they share with all life in the universe. So what are the most fundamental features of terrestrial life?

\section{The Most Fundamental Features of Terrestrial Life}

The most fundamental features of terrestrial life have been discussed in some detail (e.g. Feinberg and Shapiro, 1977; Pace, 2001; Benner et al., 2004; DeDuve, 2007). These features are often used to attempt to define life (e.g. Sagan, 1970, Joyce et al., 1994; Cleland and Chyba, 2002). Here we briefly review some major fundamental features including: liquid water as the solvent, carbon as the scaffold for biochemistry, the stoichiometry of the major bioelements, the LEGO principle, homochirality, free energy from thermodynamic disequilibria and the Darwinian evolution of inheritable molecules. 


\subsection{LIQUID WATER AS SOLVENT}

Life is made of molecules dissolved in a solvent. It seems reasonable that any life would need to be based on a liquid because of the way biomolecules, atoms and ions need to move freely and interact, both in the liquid and on the liquid-solid boundary (Benner et al., 2004; Bains, 2004). In solids, molecules lack mobility. In gases, molecules lack structure.

Whether life elsewhere can be based on liquids besides water is an open question. Bains (2004) and Benner et al. (2004) have pointed out that if life can be based on other liquids, then that liquid determines what the biochemistry will be. The elements out of which water-based life is made, hydrogen, oxygen, carbon, nitrogen, phosphorus, sulfur (HOCNPS) are the most common elements in the universe (Pace, 2001; Lodders et al., 2009). These abundances do not preclude life based on other liquids and elements but they do suggest that if there is some life that is not based on liquid water, it would not be based on HOCNPS molecules and therefore would not be as abundant as water-based life. It would be limited by the availability of nutrients just as terrestrial life is limited by the low abundance and correspondingly low availability of phosphorus. Thus, most extraterrestrial life, like terrestrial life, should be based on water as a solvent. Hence, in its search for extraterrestrial life, NASA's "follows the water" (Hubbard et al., 2002) makes sense.

\subsection{CARBON AS SCAFFOLD}

Light elements are more abundant in the universe than heavier elements. This is at least a partial explanation for why life is made of lighter elements. For example, there are $\sim 20$ carbon atoms for every silicon atom in the universe. Thus, based on abundance alone, if silicon were just as likely as carbon to be the basis of life (Benner et al., 2004), there would be 20 life forms based on carbon for each life form based on silicon.

\subsection{THE STOICHIOMETRY OF THE MAJOR BIOELEMENTS: HOCNPS}

Terrestrial life is made of HOCNPS at the 98\% (wt $\%$ ) level. The bulk elements hydrogen, oxygen, carbon, and nitrogen make up 97\%. Phosphorus and sulfur make up another $1 \%$. The remaining $2 \%$ is dominated by potassium, sodium, calcium, magnesium and chlorine.

Trace elements like iron, copper and cobalt make up $\sim 0.03 \%$ (Chopra et al., 2010). Another way to look at the chemical composition of life is as follows. Life is made of about $70 \%$ water. If we remove that $70 \%$, the rest is $65 \%$ carbon, $10 \%$ oxygen, $7 \%$ hydrogen and $7 \%$ nitrogen. These stoichiometric percentages are fairly stable and common to all life on Earth, much as Redfield ratios $\mathrm{C}: \mathrm{N}: \mathrm{P} \approx 106: 16: 1$ (Redfield, 1934) are fairly stable among phytoplankton and more generally marine biomass.

Terrestrial life's Redfield ratios (based on bacteria and humans) are approximately 106:13:2 (slightly less $\mathrm{N}$ and twice as much P as Redfield's marine biomass) (Chopra et al., 2010). Our educated guess is that if life elsewhere, is based on water, then it too will be made of the most abundant elements in the universe, HOCNPS and possibly with stoichiometric ratios that correlate strongly with the cosmic (i.e. solar) ratios H:O:C:N:P:S $\approx 430000: 230: 106: 31: 0.1: 6$ (Lodders et al., 2009). 


\subsection{THE LEGO PRINCIPLE}

Since the elemental ingredients for life are the most common elements in the universe, it is not surprising that the molecular ingredients of life are common. Terrestrial life is built of subunits called monomers such as amino acids, fatty acids, sugars and nitrogenous bases. Amino acids link together to form proteins. Fatty acids link together to form lipids. Sugars link to form carbohydrates. And nitrogenous bases combine with sugar and phosphate to make nucleotide monomers, which link together to form RNA/DNA. Life links available monomers together to make polymers, much as we link these words together to make these sentences. McKay (2004) has dubbed this monomer-linkage-strategy the "LEGO Principle". Importantly, life does not make all possible polymers. From a much larger pool of possibilities, life links a very limited set of specific monomers together to produce a very specific set of polymers. For example, out of the $\sim 100$ amino acids found in the Murchison meteorites, only 8 are part of the 20 protein amino acids that life on Earth is made of (Schmitt-Kopplin et al., 2010).

McKay (2004) and Davies et al. (2009) pointed out that abiotic processes usually produce a broad spectrum of complex molecules, while life produces a specific set of molecular polymers that shows up like a fingerprint in a molecular mass spectrometer. Since the LEGO principle is common to all terrestrial life, it may be common to all life. This is one of the biosignatures that upcoming missions to Mars will be looking for.

\subsection{HOMOCHIRALITY}

Take a dozen children, each speaking a different language and put them in a room. After a while, they will start speaking the same language. Or take a country in which horse drawn carts travel down the middle of the roads. Add more traffic and maybe a few cars. After a while, people will be driving on the right side or the left side of roads. The symmetry is broken by the necessity to get along - by the necessity to choose one side only. It does not matter which side, as long as there is general agreement. When such correlated behavior is adaptive, statistical fluctuations can get amplified into a consensus.

In terrestrial biology, the polypeptide backbones of proteins are made exclusively from homochiral (L) amino acids. Carbohydrates and nucleic acids are made with homochiral (D) sugars. Amino acids and sugars produced abiotically are usually racemic (see however Pizzarello, 2007). This distinction between racemic abiotically produced molecules and non-racemic biotically-produced molecules makes homochirality a fundamental feature of terrestrial life, and one of our expectations about life elsewhere. If aliens have roads and cars, they need agreement on which side to drive on, but there would be no way to guess whether it is on the right or left. Similarly, if extraterrestrial life is made of chiral molecules, an educated guess would be that these molecules are homochiral, but there is no way to guess whether their monomers will be $\mathrm{L}$ or $\mathrm{D}$. 


\subsection{FREE ENERGY FROM THERMODYNAMIC DISEQUILIBRIA}

The observation of planetary atmospheres out of chemical equilibrium has been proposed as a way to distinguish live planets from dead planets (Lovelock, 1975; Catling and Bergsman, 2010). Life needs to do something for a living and this living depends on extracting free energy from an environment out of thermodynamic equilibrium. This extraction is based on absorbing photons and catalyzing redox reactions (Kleidon, 2010).

If we generalize the traditional definitions of life to include all far-fromequilibrium dissipative structures (Schneider and Sagan, 2005; Lineweaver 2006, Lineweaver and Egan, 2008), then stars, volcanoes, hurricanes, convection cells and fires would be considered forms of life. Stars are based on a nuclear disequilibrium, volcanoes and hurricanes are based on a pressure and thermal disequilibrium, convection cells are based on thermal disequilibrium and fires are based on a chemical disequilibrium. Thus, some kind of disequilibrium is a common feature of all life, even with the most general definition of life.

\subsection{DARWINIAN EVOLUTION OF INHERITABLE MOLECULES}

Life has been defined as "a chemical system capable of Darwinian evolution" (Joyce 1994). All terrestrial life forms (including viruses) have this in common. If we accept this as a definition, then tautologically, extraterrestrial life will be capable of Darwinian evolution. However, to identify Darwinian evolution in extraterrestrial life, we need to identify the channel of inheritance.

None of the far-from-equilibrium-dissipative-structures listed above seem to have channels of inheritance, in which information is passed from one generation to the next. One could argue that hurricanes may be influenced by temperature, moisture and pressure gradients set-up in the tracks of previous hurricanes but there is no identifiable unit of information that is built upon through generations of hurricanes. We expect extraterrestrial life to have some form of inheritance since otherwise, we would not consider it alive. 


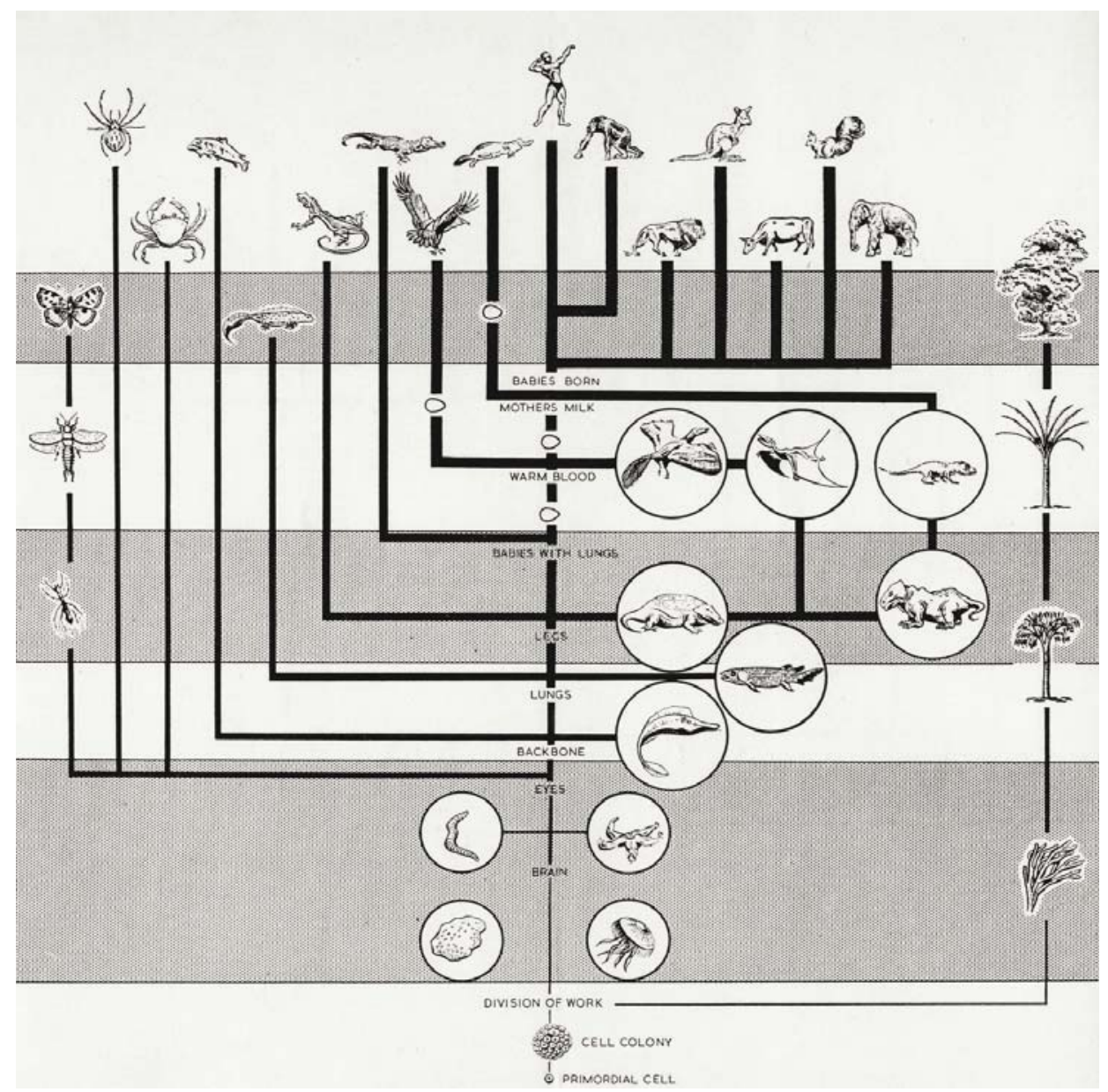

Figure 1. The Schwarzeneggerization of Life. Illustration from Gatland and Dempster (1957) in which some of the transitions that led to a male Caucasian Homo sapiens body-builder are portrayed as major transitions central to the trends of evolution. Plants are marginalized to the far right. Fungi and prokaryotes are left out. Jellyfish and star fish appear in the central lower right of the diagram, but for some reason, do not evolve to the present day. For a detailed discussion of what is wrong with such "Great Chain of Being" illustrations, see Gould (1989).

In the proto-biological RNA world (Gilbert, 1986; Joyce, 2002), bits of RNA stuck to each other. Some combinations were stable and survived while others fell apart. Strands of RNA, ribozymes, catalyzed reactions and acted as a template for selfreplication (Cech, 1985; Chen et al., 2007; Orgel, 2004). Inexact copying, point mutations and random conjugation were the source of variations and these variations were inheritable. The correlated survival of some genes and not others, and their isolation in cells, was the beginning of Darwinian evolution and life on Earth (e.g. Martin and Russell, 2003). Thus, we can expect extraterrestrial life to have inheritable molecules. 

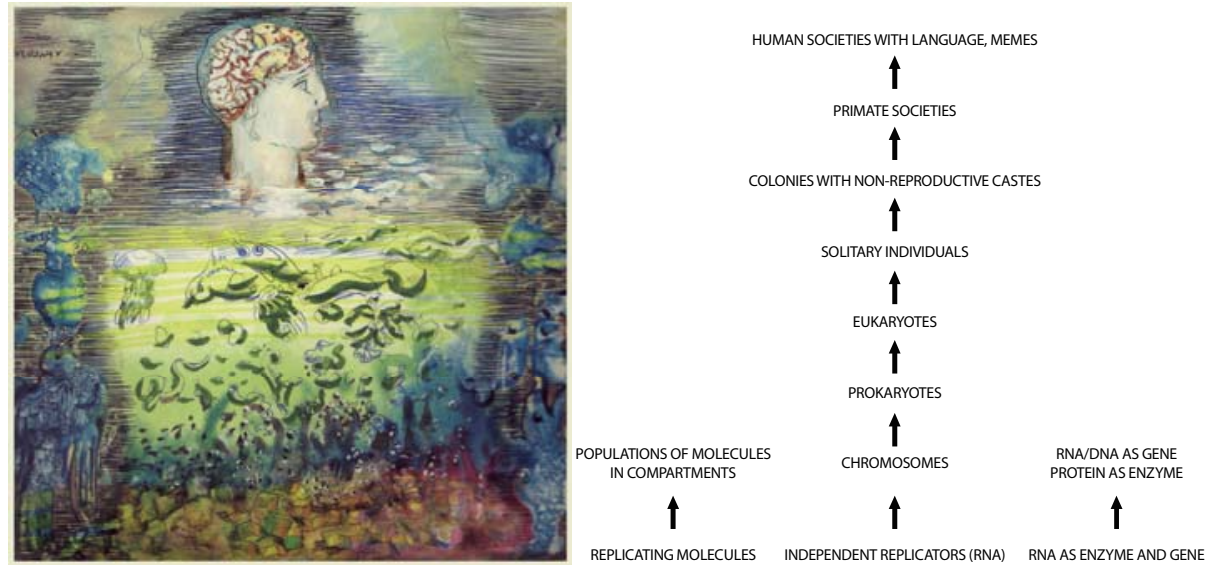

Figure 2. The Macedonification of Life. Illustration from the cover of Smith and Szathmary (1995) "The Major Transitions in Evolution" in which some of the transitions that led to a skinless Macedonian brain are portrayed as major transitions central to the evolution of life. The major transitions of life described in the book are shown on the right. Unlike Figure 1, where Schwarzenegger shares the spotlight with a dozen other creatures, the skinless Macedonian brain is alone at the top.

\section{Evolutionary transitions: early and generic vs. late and quirky}

The phylogenetic tree of life has many branches. Every extant species sits at the end of a branch - an evolutionary pathway - that one can follow back in time (Dawkins, 2004). If we follow the branches back in time, they converge two at a time into thicker branches which meet at the common trunk of the tree - the common origin of all extant life, known as the Last Universal Common Ancestor (LUCA). The fundamental features common to all life on Earth have their origins in the roots and trunk of the tree. Following the trunk and branches forward in time, many familiar species were identical (in the trunk) for the first few billion years and then recently, they diverged into different branches. For example, animals and fungi diverged about a billion years ago after sharing the same evolutionary pathway for $\sim 3$ billion years. Vertebrates and invertebrates diverged about 500 million years ago after being identical for $\sim 3.5$ billion years. Because of this pattern of divergence, the most fundamental "major transitions" are the earliest transitions - the ones that occurred in the trunk and are common to all extant terrestrial life.

Some features that are not common to all terrestrial life are often interpreted as "major transitions" in evolution (Smith and Szathmary, 1995), and are sometimes thought to be so universally adaptive that we should expect these transitions to have occurred in the evolution of extraterrestrial life. Eukaryogenesis, multicellularity, sexual reproduction and encephalization are among these. These candidates for being features of extraterrestrial life, are less secure because they are based on subjective notions of universal fitness, not on features common to all terrestrial life (Lineweaver 2005, 2009).

"Major transitions" (particularly recent ones) are arbitrary because a series of major transitions can be identified along the evolutionary pathway to any extant species. Evolution is path-dependent. There is no linear progression. Figure 1 is a misleading diagram showing how seductive the linear-progression-Great-Chain-ofBeing interpretation of evolution can be. However, Figure 2, the front cover of a 
more academically respectable book, "The Major Transitions in Evolution" by Smith and Szathmary (1995), shows much the same thing. On the right of Figure 2, the arrows summarize the major transitions that Smith and Szathmary (1995) have identified. Gould (1989) has written a concise criticism of what is wrong with such subjective interpretations of biological evolution.

Social insects do not need big brains. Moles do not need eyes. Legs are not a major transition on your way to becoming a tuna or a sequoia tree. Vertebrae are not a major transition on the evolutionary path to worms. And multicellularity and sexual reproduction are not major transitions in the evolutionary path to the marine bacterium Pelagibacter, the most abundant organism on Earth (Morris et al., 2002).

The major transitions that led to any particular terrestrial species (e.g. Homo sapiens) have no objective claim to being major transitions in the evolution of extraterrestrial life. We may consider the transition from a primate society to our specific human societies as a major transition for us, but this was not a major transition in the evolutionary paths that let to macaques, gibbons or chimps. Smith and Szathmary's list of major transitions would be appropriate for a bird except that the transition from "walking" to "flying" would replace the "primate societies" to "human societies" transition.

If we can make guesses about how terrestrial life transitioned, then we have some idea of what it transitioned from earlier on in its history. The earliest transitions of terrestrial life inform our best guesses at the nature of extraterrestrial life. On this reasoning, we suggest that extraterrestrial life had an emergence similar to our emergence in an RNA world - a viral world of replicating inheritable molecules, molecules like RNA ribozymes, both enzymes and genes, functioning in metabolism and passing on information to the next generation. These are the features listed at the bottom right of Figure 2, before any transitions take place. With regard to an extraterrestrial RNA world, the "RNA" can be thought of as any inheritable molecule.

\section{Hyperthermophilia and the Deepest Branches of Terrestrial Life}

The deepest roots of the 16s rRNA phylogenetic tree are hyperthermophilic (Fig. 3, see also Lineweaver and Schwartzman, 2005; Wong et al., 2007). Extant organisms with the shortest branches are hyperthermophiles able to tolerate temperatures above $90^{\circ} \mathrm{C}$. These organisms - Aquifex, Thermotoga, Nanoarchaeota and Korarchaeota - seem to be the best representatives of the Last Universal Common Ancestor of all terrestrial life. Hyperthermophilic organisms closest to the root suggest that LUCA was hyperthermophilic. And by extension that the origin of life on Earth was hyperthermophilic. 


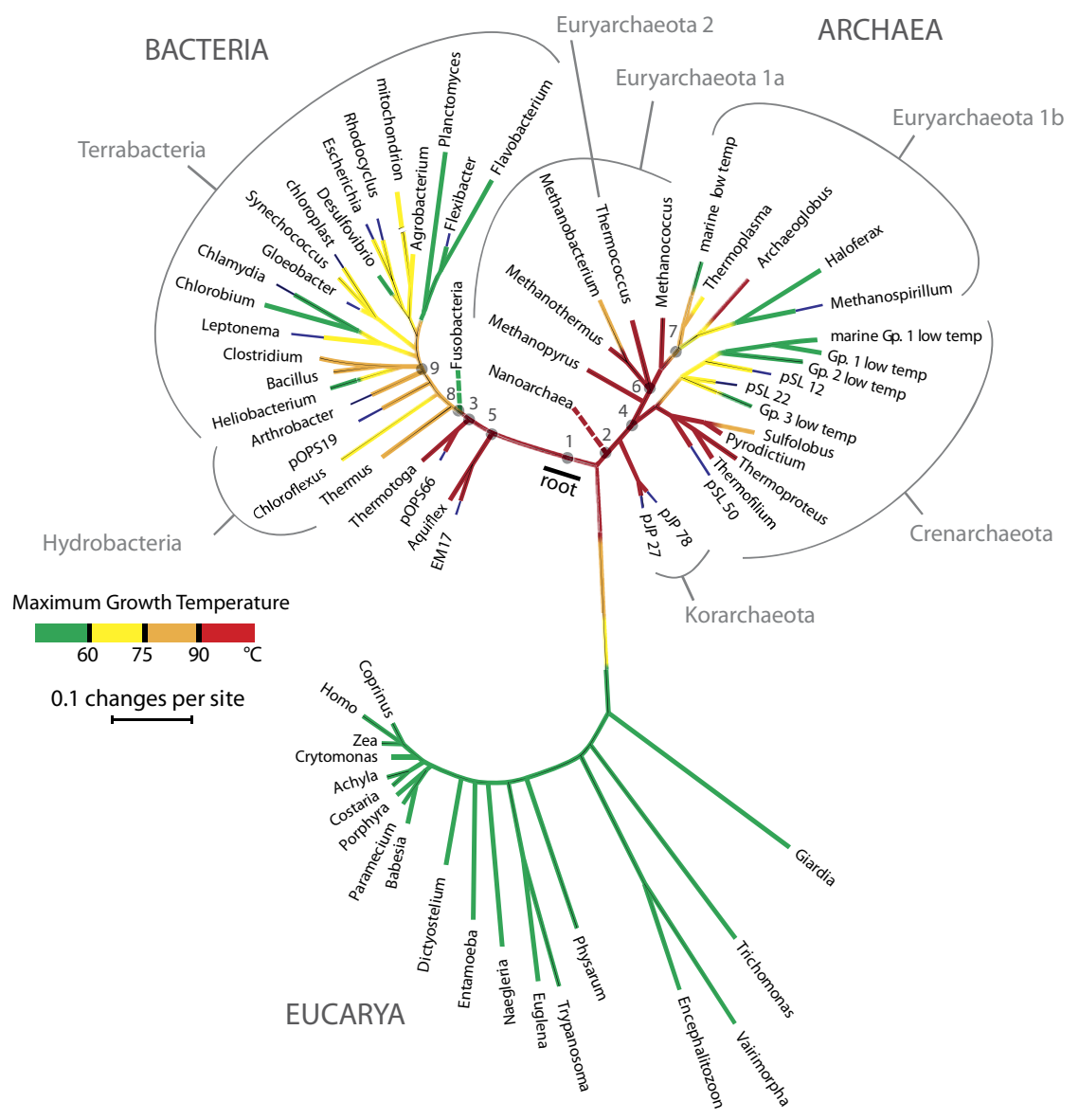

Figure 3. The 16S rRNA tree of life suggests that life started out hyperthermophilic (red) and then learned how to live at lower temperatures too. The color coding refers to the maximum temperature able to be tolerated by a given clade. For example, eukaryotes all require temperatures less than $60^{\circ} \mathrm{C}$. Plants, animals and fungi are represented in the lower left by Zea (Corn), Homo (humans) and Coprinus (mushrooms) respectively. The position of the root is from ancient gene duplication studies (Iwabe et al., 1989; Brown and Doolittle, 1995; Wong et al., 2007; Gaucher et al., 2010). Numbered nodes are described in Figure 4 and Table 1. Figure modified from Lineweaver and Schwartzman (2005) modified from Pace (1997).

Although hyperthermophilia is not now a feature common to all terrestrial life, it may have been $\sim 4$ billion years ago. Thus, we might expect extraterrestrial life to have originated as a hyperthermophilic RNA world. What it evolved into from there is much less certain and quirky. However, life everywhere may still be embedded in its origin, much as we are still embedded in our viral (RNA) world. 


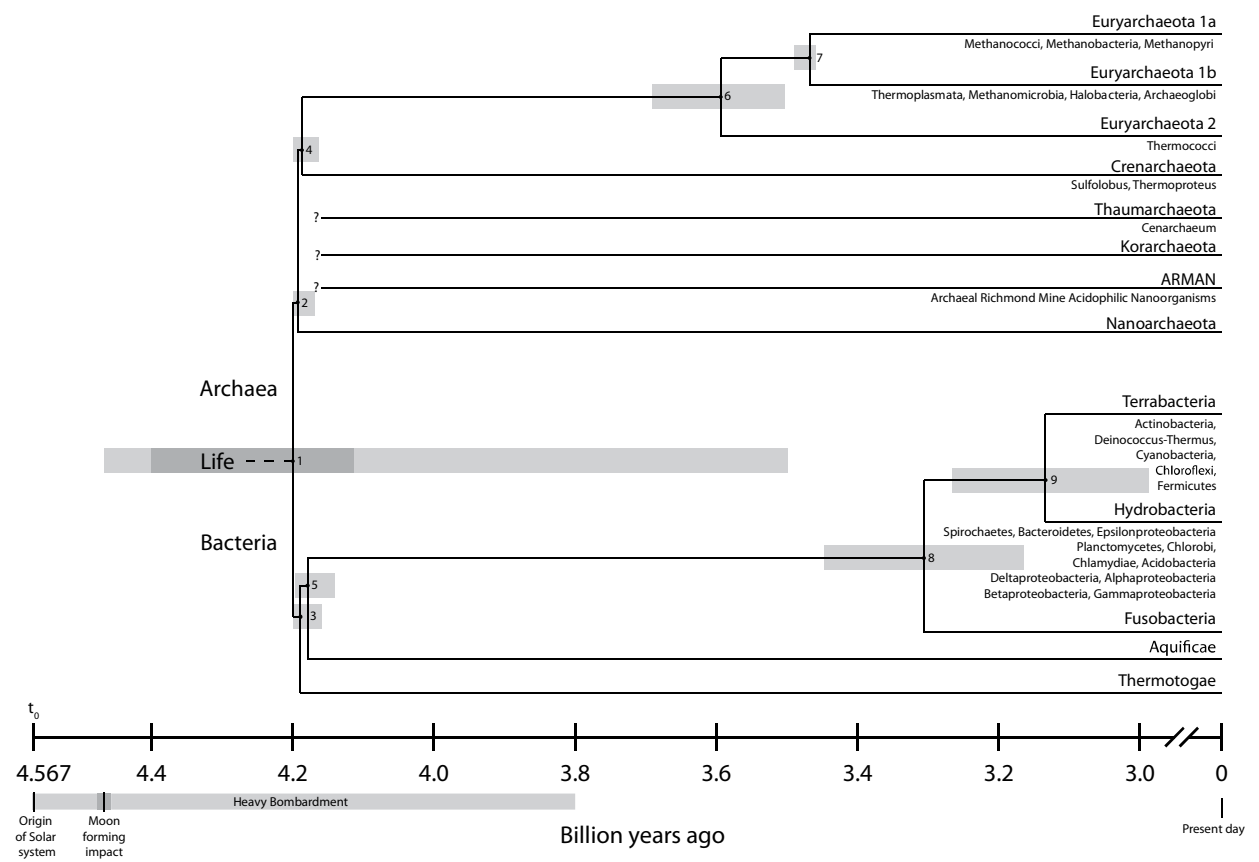

Figure 4. The earliest divergences in the phylogenetic tree of life. The first 5 divergences (nodes 1-5) are shown here at $\sim 4.2$ Gya, with overlapping relative uncertainties given by the grey horizontal bars at each node. The larger absolute uncertainties are shown for node 1 only. That is, the whole tree can shift right and left relative to the time axis at the bottom by the amount shown by the horizontal bar at node 1 .

TABLE 1. Data for Figure 4.

\begin{tabular}{|c|c|c|c|c|c|}
\hline Node & $\begin{array}{l}\text { Time } \\
\text { Mya }\end{array}$ & Lineage A & Lineage B & $\begin{array}{l}\text { Range } \\
\text { Mya }\end{array}$ & Reference \\
\hline 1 & 4200 & Bacteria & Archaea & 4400 - $4112 *$ & Hedges, '09 \\
\hline \multicolumn{6}{|l|}{ Archaea } \\
\hline 2 & 4193 & Nanoarchaeota & $\begin{array}{c}\text { Crenarchaeota \& } \\
\text { Euryarchaeota }\end{array}$ & $4200-4176$ & \multirow{4}{*}{$\begin{array}{l}\text { Battistuzzi } \\
\text { and Hedges, } \\
\text { 2009a }\end{array}$} \\
\hline 4 & 4187 & Crenarchaeota & Euryarchaeota & 4199 - 4163 & \\
\hline 6 & 3594 & Euryarchaeota2 & Euryarchaeota1 & $3691-3503$ & \\
\hline \multicolumn{3}{|c|}{ Bacteria } & Euryarchaeota1b & $3490-3460$ & \\
\hline 3 & 4189 & Thermotogae & $\begin{array}{c}\text { Aquificae \& } \\
\text { Fuso. \& Hydro. \& Terra. }\end{array}$ & $4200-4159$ & \multirow{4}{*}{$\begin{array}{l}\text { Battistuzzi } \\
\text { and Hedges, } \\
2009 b\end{array}$} \\
\hline 5 & 4179 & Aquificae & Fuso. \& Hydro. \& Terra. & $4197-4141$ & \\
\hline 8 & 3306 & Fusobacteria & Hydro. \& Terrabacteria & $3447-3165$ & \\
\hline 9 & 3134 & Hydrobacteria & Terrabacteria & $3265-2987$ & \\
\hline
\end{tabular}

*This range is the dark grey, relative uncertainty (Hedges, 2009). The larger, light grey absolute uncertainty is based on the Moon-forming impact at 4470 Mya (Halliday, 2008; Sleep et al., 1989) and the earliest fossil evidence for life $\sim 3500$ Mya (Hedges, 2009). Origin of solar system: $4567 \pm 2$ Mya (Amelin and Krot 2007). 


\section{Terrestrial ubiquity as a Frozen Accident uninformative of ET}

We have argued that universal features of life are our best guess for the nature of extraterrestrial life. However, just because a feature is universal in terrestrial life, does not necessarily mean that it is a feature of extraterrestrial life. Ubiquity among extant terrestrial life forms does not necessarily imply ubiquity among all terrestrial life that has ever lived, or among all extraterrestrial life. The tree of life has been pruned by both selection pressure and random events.

Most species that have ever existed are extinct. It is possible that a large percentage of phyla, kingdoms and even domains of life that have ever existed are extinct (e.g. Davies and Lineweaver 2005). Thus, whatever is common to all life today could be the result of a severe and possibly arbitrary pruning of the tree of life - a pruning based on fitness, but also on luck.

The case of DNA is illustrative. DNA is common to all terrestrial life forms. But the code for translating base pairs into amino acids is largely arbitrary (e.g. Tlusty, 2010; Freeland et al., 2000). That arbitrariness is what makes it an abstract symbolic code rather than a model or a pictograph system.

Crick (1968) suggested that the genetic code was universal in all organisms, and the result of a "frozen accident", unable to evolve further even if the current state were suboptimal. Frozen accidents, even when common to all extant and extinct life, would not be expected to be common to all extraterrestrial life.

\section{Summary}

Terrestrial life can be seen as a model organism representing extraterrestrial life. We have briefly reviewed some of the fundamental features common to all terrestrial life including: liquid water as the solvent, carbon as the scaffold for biochemistry, the stoichiometry of the major bioelements, the LEGO principle, homochirality, free energy from thermodynamic disequilibria and the Darwinian evolution of inheritable molecules. We have argued that our best guesses for the features of extraterrestrial life are a subset of the features on this list. Other frequently espoused candidate features (e.g. multicellularity, sexual reproduction, heads) are less secure because they are based on subjective notions of universal fitness.

By identifying the more deterministic processes that led to the origin and evolution of life on Earth and by tracking the earliest divergences, we are identifying the possibly universal processes which may have led to life elsewhere. One prediction of this reasoning is that the life forms on other worlds will have hyperthermophilic roots and will still be embedded in their earliest ancestors, much as we are still embedded in our viral (RNA) world.

\section{References}

Amelin, Y. and Krot, A. (2007) Pb isotopic age of the Allende chondrules, Meteoritics and Planetary Science, 42: 1321-1335.

Bains, W. (2004), Many Chemistries Could be Used to Build Living Systems, Astrobiology, 4: 137-167

Battistuzzi, F.U. and Hedges, S.B. (2009a) Archaebacteria, In: S. B. Hedges and S. Kumar (eds.) The Timetree of Life. Oxford University Press, pp. 101-105.

Battistuzzi, F.U. and Hedges, S.B. (2009b) Eubacteria, In: S. B. Hedges and S. Kumar (eds.) The Timetree of Life. Oxford University Press, pp. 106-115. 
Benner, S.A., Ricardo, A., and Carrigan, M.A. (2004) Is there a common chemical model for life in the universe?, Current Opinion in Chemical Biology, 8: 672-689

Brown, J.R. and Doolittle, W.F. (1995) Root of the universal tree of life base on ancient aminoacyltRNA synthetase gene duplications, PNAS, 92: 2441-2445

Catling, D.C. and Bergsman, D.S. (2010) On detecting exoplanet biospheres from atmospheric chemical disequilibrium, Astrobiology Science Conference 2010 Abstract \#5533.

Cech, T. R. (1985) Self-Splicing RNA: Implications for Evolution, In: G.H. Bourne, J.F. Danielli and K.W. Jeon, (eds.), International Review of Cytology, Academic Press, 93: 3-22

Chen, X., Li, N. and Ellington, A. (2007), Ribozyme Catalysis of Metabolism in the RNA World. Chemistry \& Biodiversity, 4: 633-655

Chopra, A., Lineweaver, C.H., Brocks, J.J. and Ireland, T.R. (2010) Palaeoecophylostoichiometrics: Searching for the Elemental Composition of the Last Universal Common Ancestor, In: W. Short and I. Cairns (eds.) Australian Space Science Conference Series: 9th Conference Proceedings. NSSA Full Referreed Proceedings CD, National Space Society of Australia Ltd.

Cleland, C. and Chyba, C. (2002) Defining Life, Origins Life Evol. Biosphere, 32: 387-393

Crick, F. H. C. (1968) The origin of the genetic code, J. Mol. Biol. 38: 367-379

Davies, P.C.W. and Lineweaver, C.H. (2005) Finding a Second Sample of Life on Earth, Astrobiology, 5: $154-163$

Davies, P.C.W., Benner, S.A., Cleland, C.E., Lineweaver, C.H., McKay, C.P. and Wolfe-Simon, F. (2009) Signatures of a Shadow Biosphere, Astrobiology, 9: 241-249

Dawkins, R. (2004) The Ancestor's Tale: a Pilgrimage to the Dawn of Life, Weidenfeld and Nicholson, London

De Duve, C. (1995) Vital Dust: The Origin and Evolution of Life on Earth, Basic Books, New York

De Duve, C. (2007) Chemistry and Selection, Chemistry and Diversity, 4: 574-583

Feinberg, G. and Shapiro, R. (1980) Life Beyond Earth: the Intelligent Earthling's Guide to Life in the Universe, William Morrow, New York

Freeland, S.J., Knight, R.D., Landweber, L.F. and Hurst, L. D. (2000) Early Fixation of an Optimal Genetic Code, Molecular Biology and Evolution, 17: 511-518

Gatland, K.W. and Dempster, D.D. (1957) The Inhabited Universe: an enquiry staged on the frontiers of knowledge, McKay, NY

Gaucher, E. A., Kratzer, J. T. and Randall, R. N. Deep (2010) Phylogeny - How a Tree Can Help Characterize Early Life on Earth, Cold Spring Harbor Perspectives in Biology, 2:a002238

Gilbert, W. (1986) Origin of life: The RNA world, Nature, 319: 618

Gould, S.J. (1977) Ontogeny and Phylogeny, Harvard Univ. Press

Gould, S.J. (1989) Wonderful Life: The Burgess Shale and the Nature of History, Chapter 1: Implications of an Iconography, Norton \& Company, NY

Gould, S. J. (2002) The Structure of Evolutionary Theory, Harvard Univ. Press

Halliday, A. N. (2008) A young Moon-forming giant impact at 70-110 million years accompanied by late-stage mixing, core formation and degassing of the Earth, Phil. Trans. Roy. Soc. A, 366: 41634181

Hedges, S.B. (2009) Life, In: S. B. Hedges and S. Kumar (eds.) The Timetree of Life. Oxford University Press, pp. 89-98.

Hubbard, G. S., Naderi, F.M. and Garvin, J.B. (2002) Following the water, the new program for Mars exploration. Acta Astron., 51: 337-350

Ida, S. and Lin, D.N.C. (2004) Toward a Deterministic Model of Planetary Formation. I. A Desert in the Mass and Semimajor Axis Distributions of Extrasolar Planets, ApJ, 604: 388-413

Iwabe, N., Kuma, K.-I., Hasegawa, M., Osawa, S. and Miyata, T. (1989) Evolutionary relationship of archaebacteria, eubacteria, and eukaryotes inferred from phylogenetic trees of duplicated genes, PNAS, 86: 9355-9359

Joyce, G.F. (2002) The antiquity of RNA based evolution. Nature Insight, 418: 214-221

Joyce, G.F. (1994) Foreword In: D.W. Deamer and G.R. Fleischacker (eds.) Origins of Life: The Central Concepts, Jones and Bartlett Publishers, Boston, pp. xi-xii.

Kleidon, A. (2010) Life, Hierarchy, and the Thermodynamic Machinery of Planet Earth, Physics of Life Reviews, (in press)

Kuchner, M.J. (2003) Volatile-rich Earth-Mass Planets in the Habitable Zone, ApJ, 596: L105-L108

Léger, A., Selsis, F., Sotin, C. et al. (2004) A new family of planets? “Ocean-Planets”, Icarus, 169: 499504

Lineweaver, C.H. (2005) Intelligent Life in the Universe Book Review of "Intelligent Life in the Universe: From Common Origins to the Future of Humanity" by Peter Ulmschneider, review published in Astrobiology, 5: 658-661

Lineweaver, C.H. (2006) We have not detected extraterrestrial life, or have we? In: J. Seckbach and M. Walsh (eds.) Life as we know it: Cellular Origins and Life in Extreme Habitats and Astrobiology, Springer Life Sciences, Dordrecht, The Netherlands, p.445 
Lineweaver, C.H. (2009) Paleontological Tests: Human-like Intelligence is not a Convergent Feature of Evolution, In: J. Seckbach and M. Walsh (eds.) From Fossils to Astrobiology, Cellular Origins and Life in Extreme Habitats and Astrobiology, Springer, 12: 353-368

Lineweaver, C.H. and Egan, C. (2008) Life, Gravity and the Second Law of Thermodynamics, Physics of Life Reviews, 5: 225-242

Lineweaver C.H., Grether, D. (2003) What Fraction of Sun-like Stars have Planets?, ApJ, 598: 13501360

Lineweaver, C.H. and Schwartzman, (2005) Cosmic Thermobiology: Thermal Constraints on the Origin and Evolution of Life in the Universe, In: J. Seckbach (ed.) Origins: Cellular Origins and Life in Extreme Habitats and Astrobiology, Springer, 6: 233-248

Lodders, K., Palme, H. and Gail, H. -P. (2009) Abundances of the elements in the solar system, LandoltBornstein, New Series, Astronomy and Astrophysics, Springer Verlag, Berlin Volume VI/4B, Chapter 4.4, J.E. Trumper (ed), pp 560-630

Lovelock, J.E. (1975) Thermodynamics and the recognition of alien biospheres. Proc. Roy. Soc. London B., 189: 167-181

Martin, W. and Russell, M.J. (2003) On the origins of cells: a hypothesis for the evolutionary transitions from abiotic geochemistry to chemoautotrophic prokaryotes, and from prokaryotes to nucleated cells, Phil. Trans. R. Soc. Lond. B Biol. Sci. 358: 59-83

McKay, C. P. (2004) What is life - and how do we search for it on other worlds? PLOS Biol. 2: 1260 1263

McShea, D.W. and Brandon, R.N. (2010) Biology's First Law: The Tendency for Diversity and Complexity to Increase in Evolutionary Systems, Univ. Chicago Press.

Mordasini, C., Alibert, Y., Benz, W. and Naef, D. (2009) Extrasolar planet population synthesis. II. Statistical comparison with observations, Astronomy and Astrophysics, 501: 1161-1184

Morris, R. M., Rappe, M. S., Connon, S. A., Vergin, K. L., Siebold, W. A., Carlson, C. A. and Giovannoni, S. J. (2002) SAR11 clade dominates ocean surface bacterioplankton communities Nature, 420:806-810

Orgel, L.E. (2004) Prebiotic Chemistry and the Origin of the RNA World, Crit. Rev. Biochem. Mol. Biol. 39: 99-123

Pace, N. (1997) A Molecular View of Microbial Diversity and the Biosphere, Science, 276: 734-740

Pace, N. (2001) The universal Nature of Biochemistry, PNAS, 98: 805-808.

Pizzarello, S. (2007) The Chemistry that Preceded Life's Origin: A Study Guide from Meteorites, Chemistry and Biodiversity, 4: 680-693

Redfield A.C. (1934) On the proportions of organic derivations in sea water and their relation to the composition of plankton, In: R.J. Daniel James (ed.) Johnstone Memorial Volume. University Press of Liverpool, pp. 177-192

Robles, J.A., Lineweaver, C.H., Grether, D. et al. (2008) A Comprehensive Comparison of the Sun to other Stars: searching for self-selection effects, ApJ, 684:691-706

Sagan, C. (1970) "Life" in The Encyclopedia Britannica, W. Benton, London, 14th Edition.

Schmitt-Kopplin, P., Gabelica, Z., Gougeon, R. D. et al. (2010) High molecular diversity of extraterrestrial organic matter in Murchison meteorite revealed 40 years after its fall, PNAS, 107: 2763-2768

Schneider, E.D. and Sagan, D. (2005) Into the Cool: Energy Flow, Thermodynamics, and Life. The University of Chicago Press, Chicago and London

Sleep, N.H., K. J. Zanhnle, J. F. Kasting and H. J. Morowitz, (1989) Annihilation of ecosystems by large asteroid impacts on the early Earth, Nature, 342: 139-142

Smith, J.M. and Szathmáry, E. (1995) The Major Transitions in Evolution, Oxford Univ. Press, Oxford

Tlusty, T. (2010) A colorful origin for the genetic code: Information theory, statistical mechanics and the emergence of molecular codes, Physics of Life Reviews, 7: 362-376

Wong, J.T-F., Chen, J., Mat, W-K., Ng, S-K., Xue, H. (2007) Polyphasic evidence delineating the root of life and roots of biological domains, Gene, 403: 39-52 